\title{
Targeting survivin using a combination of miR-494 and survivin shRNA has synergistic effects on the suppression of prostate cancer growth
}

\author{
JIN ZHU $^{1 *}$, CHENWEN SUN ${ }^{1-3^{*}}$, LIPING WANG ${ }^{1,4^{*}}$, MING XU $^{1}$, YACHEN ZANG $^{1}$, YIBIN ZHOU $^{1}$, \\ XIAOLONG LIU ${ }^{1}$, WEI TAO ${ }^{1}$, BOXIN XUE ${ }^{1}$, YUXI SHAN ${ }^{1}$ and DONGRONG YANG ${ }^{1}$ \\ ${ }^{1}$ Department of Urology, The Second Affiliated Hospital of Soochow University, Suzhou, Jiangsu 215004; \\ ${ }^{2}$ Department of Urology, Affiliated Hospital of Jiangnan University; ${ }^{3}$ Department of Urology, \\ Wuxi Fourth People's Hospital, Wuxi, Jiangsu 214062; ${ }^{4}$ Department of Urology, \\ Yancheng Third People's Hospital, Yancheng, Jiangsu 224001, P.R. China
}

Received February 7, 2015; Accepted November 30, 2015

DOI: $10.3892 / \mathrm{mmr} .2015 .4739$

\begin{abstract}
Castration-resistant prostate cancer (CRPC) remains an obstacle in the current treatment provided for prostate cancer (PCa). Survivin, an apoptosis inhibitor, has been found to be involved in the progression of $\mathrm{PCa}$, and is a promising candidate target for CRPC therapy. Micro (mi) RNAs are involved in the progression of $\mathrm{PCa}$ through the regulation of multiple genes. One of the objectives of the present study was to investigate the effect of miRNA (miR)-494 on the expression of survivin, as well as on PCa growth. The present study also aimed to assess whether co-transfecting miR-494 with survivin short hairpin (sh)RNA has synergistic effects on suppressing PCa proliferation or the expression of survivin. Gene Expression Omnibus datasets with clinical PCa miRNA expression profiles were utilized to analysis the expression of miR-494 in Ca, compared with normal prostate samples. PC3 cells, a CRPC cell line, were transfected with either an miR-494 expression adenovius, a survivin shRNA adenovirus or the two together, to examine their effect on PCa growth and the expression of survivin in vitro and in vivo. miR-494 was downregulated in PCa tissue samples and in the PC-3 cell line. miR-494 targeted survivin at the translational level in PCa. Overexpression of miR-494 and silencing survivin RNA through the use of survivin shRNA inhibited the expression of survivin and attenuated PC-3 cell growth in vitro and in vivo. Notably, co-transfecting miR-494 with survivin shRNA had synergistic effects on suppressing prostate cancer proliferation
\end{abstract}

Correspondence to: Dr Dongrong Yang, Department of Urology, The Second Affiliated Hospital of Soochow University, 1055 Sanxiang Road, Suzhou, Jiangsu 215004, P.R. China

E-mail: doc_ydr@hotmail.com

${ }^{*}$ Contributed equally

Key words: prostate cancer, microRNA-494, survivin via further suppression of the expression of survivin. These results suggested that using multiple methods to inhibit the function of survivin may have improved efficacy for treating PCa.

\section{Introduction}

Prostate cancer ( $\mathrm{PCa}$ ) is the most commonly diagnosed malignancy and the second leading cause of cancer-associated mortality in men in America and England. (1) In Asia, the incidence and morbidity rates of PCa have increased markedly in previous decades. However, a large proportion of new cases are at an advanced stage at the time of diagnosis. Androgen deprivation therapy (ADT) is the first choice of treatment, however, a major obstacle is that the sensitivity of PCa to ADT treatment is reduced over time and eventually develops into castration-resistant prostate cancer (CRPC). At present, the efficacy of other treatment options for CRPC, including chemotherapy, radiotherapy and immunotherapy is insufficient.

The mechanism causing CRPC to be refractory to current treatment options remains to be fully elucidated. It has been suggested that the possible mechanism is due to the overexpression of survivin (2-4). Survivin (baculoviral inhibitor of apoptosis protein repeat containing 5) is a member of the inhibitor of apoptosis protein (IAP) family, which has been implicated in inhibiting apoptosis and controlling mitotic progression. Previous evidence that survivin is overexpressed in the majority of types of human cancer (5-10), but absent in normal adult tissues (11) has led to the proposal of targeting survivin as a promising alternative treatment for cancer, including CRPC $(12,13)$.

MicroRNAs (miRNAs), a set of small, non-coding RNAs of 21-23 nucleotides in length, can regulate target genes via the degradation of mRNA or by suppressing translation and, consequently, acting as tumor suppressor or promoter (oncomiR) to regulate cancer initiation and progression. For example, miR-155 functions as an oncomiR in breast cancer by targeting suppressor of cytokine signaling-1 (14). miR-34a 
inhibits PCa stem cell metastasis by directly repressing cluster of differentiation 44 (15). miR-494 acts as a tumor suppressor in different types of cancers by targeting $\mathrm{B}$ cell lymphoma (Bcl)-2 interacting mediator of cell death (BIM) (16), KIT (17), and survivin (18) to inhibit cancer proliferation and metastasis.

The function of miR-494 in PCa remains to fully elucidated. In the present study investigated whether miR-494 targeted survivin and whether miR-494 and survivin shRNA in combination had a synergistic effect on the suppression of PCa growth.

\section{Materials and methods}

Cell culture. The present study was approved by the ethics committee of the Second Affiliated Hospital of Soochow University (Suzhou, China). The PC-3 human CRPC cell line and RWPE-1 human normal prostate epithelial cell line were obtained from the Chinese Academy of Science (Shanghai, China). The PC-3 cells were maintained in RPMI 1640 medium (Gibco; Thermo Fisher Scientific, Inc., Waltham, MA, USA), containing penicillin (25 U/ml; Gibco; Thermo Fisher Scientific, Inc.), streptomycin $(25 \mathrm{~g} / \mathrm{ml}$; Thermo Fisher Scientific, Inc.), 1\% L-glutamate (Thermo Fisher Scientific, Inc.), and 10\% fetal bovine serum (FBS; Thermo Fisher Scientific, Inc.), at $37^{\circ} \mathrm{C}$ with $5 \% \mathrm{CO}_{2}$ in a humidified atmosphere. The RWPE-1 cells were maintained in complete keratinocyte-serum-free medium (K-SFM), containing $50 \mu \mathrm{g} / \mathrm{ml} \mathrm{BPE}$ (Gibco; Thermo Fisher Scientific, Inc.) and $5 \mathrm{ng} / \mathrm{ml}$ EGF (Gibco; Thermo Fisher Scientific, Inc.), with penicillin $(25 \mathrm{U} / \mathrm{ml})$ and streptomycin $(25 \mathrm{~g} / \mathrm{ml})$, at $37^{\circ} \mathrm{C}$ with $5 \% \mathrm{CO}_{2}$ in a humidified atmosphere.

Plasmids and adenovirus. Human genomic DNA (Promega Corporation, Madison, WI, USA) was used as template DNA. The sequence of the hsa-miR-494 genomic clone was designed, according to the information in miRbase (http://www.mirbase. org/). The region containing the miR-494 stem-loop sequence was amplified using polymerase chain reaction (PCR) and was inserted into the pDC315-EGFP plasmid for the overexpression of miR-494. An adenovirus packaging system $\left(\mathrm{AdMax}^{\mathrm{TM}}\right)$ was used to produce the adenovirus. For RNA interference (RNAi), a survivin shRNA recombined adenovirus vector was constructed and preserved in the Department of Urology, The Second Affiliated Hospital of Soochow University (Suzhou, China). When the miR-494 was co-transfected with survivin shRNA (sequence, 5'-caccggaccaccgcatctctacattcaagacgtgta gagatgcggtggtccttttttg-3'), the virus derived from each plasmid was reduced by half. The sequences of the primers used are listed in Table I, they were obtained from Sangon Biotech Co., Ltd., Shanghai, China. The cells were infected with the adenovirus with $8 \mu \mathrm{g} / \mu \mathrm{l}$ polybrene (Sigma-Aldrich, St. Louis, MO, USA) at a confluence of $60 \%$.

$R N A$ extraction and reverse transcription-quantitative $P C R$ $(R T-q P C R)$ for determination of miRNA levels. RNA was extracted from the cells using an mirVana ${ }^{\mathrm{TM}}$ miRNA Isolation kit (Ambion; Thermo Fisher Scientific, Inc.), according to the manufacturer's protocol. A total of $2 \mu \mathrm{g}$ RNA of each sample was used for the RT reaction. The subsequent qPCR was performed using miRNA-specific primers to analyze the
miRNA expression levels, with U6 snRNA used as an internal control. The SYBR FAST qPCR kit (Kapa Biosystems, Inc., Wilmington, MA, USA) was used with $10 \mu \mathrm{l}$ Master mix, $0.4 \mu \mathrm{l}$ forward primer $(10 \mu \mathrm{M})$ and $0.4 \mu \mathrm{l}$ reverse primer $(10 \mu \mathrm{M})$ and $1 \mu \mathrm{l}$ cDNA. Double distilled water $(8.2 \mu \mathrm{l})$ was added to bring the total volume to $20 \mu \mathrm{l}$. The thermocycling steps were as follows: $95^{\circ} \mathrm{C}$ for $3 \mathrm{~min}$; 45 cycles of $95^{\circ} \mathrm{C}$ for $3 \mathrm{sec}, 60^{\circ} \mathrm{C}$ for $20 \mathrm{sec}$ and $72^{\circ} \mathrm{C}$ for $1 \mathrm{sec}$; and a final extension step at $72^{\circ} \mathrm{C}$ for $5 \mathrm{~min}$. The sequences of the primers used are listed in Table I.

$R T-q P C R$. The cells were collected at a confluence of $90 \%$ and total RNA was extracted using TRIzol reagent (Invitrogen; Thermo Fisher Scientific, Inc.), and $2 \mu \mathrm{g}$ total RNA from each sample was converted into complementary DNA using a commercially available RT-qPCR kit (Promega Corporation). The resultant complementary DNAs were used in the qPCR reactions using gene-specific primers, and the products were analyzed using gel electrophoresis. The sequence of the primers used are listed in Table I.

Western blot analysis. The cells were washed with phosphate-buffered saline (PBS) and lysed in radioimmunoprecipitation assay buffer (Thermo Fisher Scientific, Inc.). Proteins $(30 \mu \mathrm{g})$ were separated by $10 \%$ SDS-PAGE (Sangon Biotech Co., Ltd.) and then transferred onto polyvinylidene difluoride membranes (EMD Millipore, Billerica, MA, USA). The membranes were blocked in 5\% non-fat milk in PBS with $0.05 \%$ Tween 20 (Thermo Fisher Scientific, Inc.) for $1 \mathrm{~h}$ at room temperature, and then incubated with diluted primary antibodies overnight at $4^{\circ} \mathrm{C}$. The antibodies were monoclonal rabbit anti-human antibodies against survivin (Cell Signaling Technology, Inc., Danvers, MA, USA; cat. no. 2808; 1:2,000) or monoclonal mouse anti-human antibodies against GAPDH (Abcam, Cambridge, MA, USA; cat. no. ab8245; $1: 5,000)$. The blots were then incubated with horseradish peroxidase-conjugated secondary antibodies for $1 \mathrm{~h}$ at room temperature, washed and developed using an enhanced chemiluminescence system (Gel Doc XR+; Bio-Rad Laboratories, Inc., Hercules, CA, USA). The secondary antibodies were horse anti-mouse and goat anti-rabbit, diluted 1:5,000 (Cell Signaling Technology, Inc.; cat. nos. 7076 and 7074, respectively). The expression levels of survivin were compared with those of $\beta$-actin for further quantitative analysis using Image $\mathbf{J}$ software, version 1.48 (National Institutes of Health, Bethesda, MD, USA).

3-(4,5-dimethylthiazol-2-yl)-2,5-diphenyltetrazolium bromide (MTT) assay. The cells were seeded in a 96-well plate at a concentration of $1 \times 10^{4}$ cells/well, and were maintained at $37^{\circ} \mathrm{C}$ for 24,48 and $72 \mathrm{~h}$ following transfection. The cells were then treated with MTT $(0.5 \mathrm{mg} / \mathrm{ml})$ for $4 \mathrm{~h}$ at $37^{\circ} \mathrm{C}$. The absorbance at $570 \mathrm{~nm}$ was determined using a microplate reader (Model 550; Bio-Rad Laboratories, Inc.).

Flow cytometric analysis. Cell cycle and apoptosis were determined using either propidium iodide (PI; BD Biosciences, Franklin Lakes, NJ, USA) staining or an Annexin V-Allophycocyanin (APC) detection kit from BD Pharmingen (San Diego, CA, USA). At $72 \mathrm{~h}$ post-transfection, the cells were washed with cold PBS, and either stained with PI or 
Table I. Primers used for cloning, qPCR and RT-qPCR.

\begin{tabular}{|c|c|c|}
\hline Primer & Sequence & Use \\
\hline hsa-miR-494 RT & 5'-gtcgtatccagtgcagggtccgaggtattcgcactggatacgacgaggtttc-3' & qPCR \\
\hline hsa-miR-494 Up & $5^{\prime}-$ gcgcgcgctgaaacatac-3' & qPCR \\
\hline hsa-miR-494 Down & 5'-gggtccgaggtattcgcact-3' & qPCR \\
\hline u6 RT & 5'-aaaatatggaacgcttcacgaatttg-3' & qPCR \\
\hline u6 Up & $5^{\prime}$-ctcgettcggcagcacatatact-3' & qPCR \\
\hline u6 Down & $5^{\prime}$-acgcttcacgaatttgcgtgtc-3' & qPCR \\
\hline hsa-mir-494-EcoRI F & $5^{\prime}-\operatorname{cggccgcgactctagttgattttttttgtttgttttttgatcagtgctaatcttcg-3^{\prime }}$ & Cloning \\
\hline hsa-mir-494-EcoRI R & 5'-ataagcttgatatcggacgcatggcacgctgtc-3' & Cloning \\
\hline survivin shRNA Up & 5'-caccggaccaccgcatctctaca ttcaagacg tgtagagatgcggtggtccttttttg-3' & Cloning \\
\hline survivin shRNA Down & 5'-agctcaaaaaaggaccaccgcatctctacacgtcttgaatgtagagatgcggtggtcc-3' & Cloning \\
\hline Survivin F & $5^{\prime}$-gcatgggtgccccgacgttg-3' & RT-qPCR \\
\hline Survivin $\mathrm{R}$ & 5'-gctccggccagaggctcaa-3' & RT-qPCR \\
\hline GAPDH F & 5'-tgatgacatcaagaaggtggtgaa-3' & RT-qPCR \\
\hline GAPDH R & 5'-tccttggaggccatgtgggec-3' & RT-qPCR \\
\hline$\beta$-actin F & $5^{\prime}$-gtccaccgcaaatgcttcta-3' & RT-PCR \\
\hline$\beta$-actin R & $5^{\prime}$-tgctgtcaccttcaccgttc-3' & RT-PCR \\
\hline
\end{tabular}

qPCR, quantitative polymerase chain reaction; RT-qPCR, reverse transcription qPCR; F, forward; R, reverse; shRNA, short hairpin RNA; miR, microRNA; RT, reverse transcriptase.

A

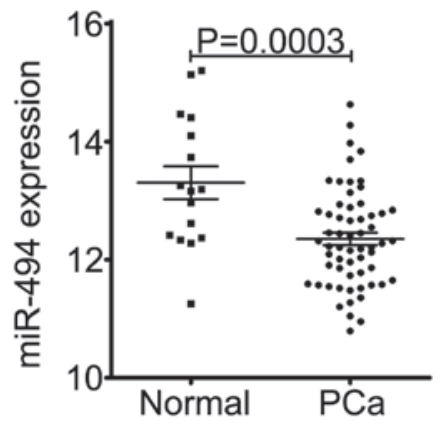

B

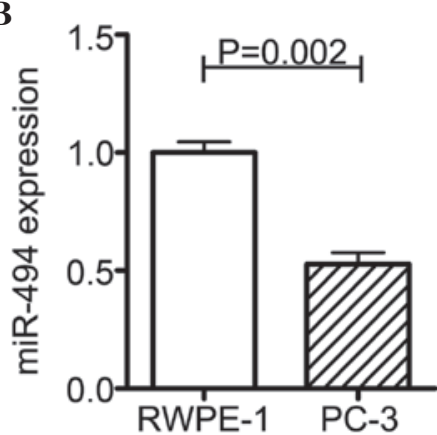

Figure 1. Expression levels of miR-494 are decreased in PCa samples and cell line. (A) Microarray data of 60 PCa samples and 16 normal prostate samples from the Gene Expression Omnibus dataset shows that the PCa tissue samples had significantly lower levels of miR-494, compared with the normal prostate tissue. (B) Expression levels of miR-494 wee lower in the PC-3 cells, compared with normal prostate RWPE-1 cells. The expression levels of miR-494 in normal and Pa cell lines were measured using quantitative polymerase chain reaction. Each sample was detected by triplicate. Values are expressed as the mean \pm standard deviation. miR, microRNA; PCa, prostate cancer.

Annexin V-APC/PI double staining, and analyzed using flow cytometry, according to the manufacturer's protocol.

Subcutaneous injection mouse model. All animal experiments were performed, according to a protocol approved by the Ethics Committee of the Second Hospital of Soochow University and in compliance with national and European regulations. A total of 25 male nude mice (age, 6-8 weeks; weight, 25-30 g) were purchased from the Experimental Animal Center of Soochow University. They were housed under standard conditions with a 12 -h light/dark cycle at $23 \pm 3^{\circ} \mathrm{C}, 55 \pm 5 \%$ relative humidity and access to food and water ad libitum. The mice were randomly assigned into the following groups, each containing five mice: PBS group; scr1 group; oe494 group; shSur group and oe494+shSur group. The pretreated PC-3 cells $\left(10^{7} /\right.$ mouse) were mixed with Matrigel (BD Biosciences) were subcutaneously injected into the right axillary space of each nude mice. When the tumors were palpable, sliding calipers were used to measure the maximum longitude diameter and transverse diameter of each tumor every 5 days. The tumor volumes were calculated according to the following formula: Volume $\left(\mathrm{cm}^{3}\right)=\mathrm{ab}^{2} / 2$. The mice were then sacrificed 55 days following injection by cervical dislocation, and tumor tissue samples were harvested and used for western blot analysis and immunohistochemical staining.

Immunohistochemistry. The tumor tissues were fixed in 4\% neutral-buffered paraformaldehyde (Sigma-Aldrich) and embedded in paraffin (Sigma-Aldrich). The primary antibodies were: monoclonal rabbit anti-human against survivin (dilution, 1:100); monoclonal rabbit anti-human against Bcl-2-associated X protein (BAX; Cell Signaling Technology, 
A

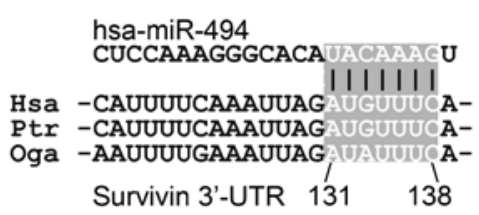

B

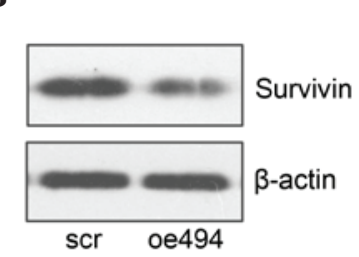

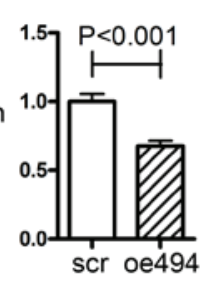

C

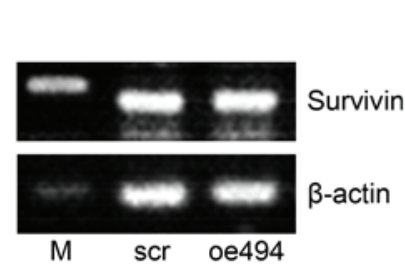

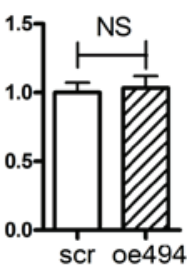

Figure 2. miR-494 targets survivin at the translational level. (A) Schematic diagram of putative miR-494 binding sites in the 3'-UTR of the survivin gene. (B) Western blot analysis revealed that overexpression of miR-494 significantly decreased the protein levels of survivin when PC-3 cells were transfected with miR-494 expression or the control adenovirus for $72 \mathrm{~h}$. (C) Reverse transcription-quantitative polymerase chain reaction analysis revealed that the mRNA expression of survivin was not affected by the overexpression of miR-494 for $72 \mathrm{~h}$. miR, microRNA; UTR, untranslated region; M, marker.

Inc.; cat. no. 5023; 1:100); monoclonal rabbit anti-human against BCL2 (Cell Signaling Technology, Inc.; cat. no. 3796; 1:100); and monoclonal rabbit anti-human against caspase 3 (Biogot Technology Co., Ltd., Nanjing, China; cat. no. BS1518; 1:100) were used for staining. Primary antibodies were recognized by goat anti-rabbit biotinylated secondary antibody (Vector Laboratories, Inc., Burlingame, CA, USA; cat. no. BA-1000; 1:1,000) and visualized using a VECTASTAIN ABC peroxidase system and peroxidase substrate DAB kit (Vector Laboratories, Inc.). Semi-quantification of the positive staining signals was performed using ImageJ software.

Statistical analysis. Values are expressed as the mean \pm standard deviation. Student's $t$-test and analysis of variance were used to determine significant differences using SPSS 19.0 (IBM SPSS, Armonk, NY, USA). P<0.05 (two-sided) was considered to indicate a statistically significant difference.

\section{Results}

Expression levels of miR-494 are lower in PCa samples and PC-3 cells. Previous studies have indicated that the expression of miR-494 is decreased in different types of cancer and acts as a tumor suppressor $(19,20)$. Therefore, the present study aimed to determine whether the expression of miR-494 in $\mathrm{PCa}$ is also lower than that in normal prostate tissue.

An NCBI Gene Expression Omnibus (http://www.ncbi. nlm.nih.giv/gds) dataset search was performed and, in the GSE8126 dataset (20), 60 PCa clinical samples and 16 normal prostate samples were collected and subjected to an miRNA-specific expression array. As expected, the expression of miR-494 was markedly lower in the PCa samples, compared with the normal prostate tissues (Fig. 1A).

The PC-3 CRPC cell line was then randomly selected, and the expression levels of miR-494 in the PC-3 cells and in the RWPE-1 normal prostate epithelial cell line were determined using RT-qPCR. The results showed that the expression of miR-494 was reduced in the PC-3 cells, compared with that in the RWPE-1 cells (Fig. 1B).

Taken together, the results (shown in Fig. 1A and B) demonstrated lower expression levels of miR-494 in PCa.

miR-494 targets survivin at the translational level. The survivin gene is a well-known oncogene, which belongs to the anti-apoptosis gene family; its expression is correlated with tumor malignancy and progression in a variety of types of cancer, as well as PCa (5-10). It has been reported that miR-494 negatively regulates the gene expression of survivin in acute myeloid leukemia cells (18), and the bioinformatics analysis performed in the present study also indicated that miR-494 regulated survivin via binding to its 3'-untranslated region (Fig. 2A). However, this effect has not yet been clarified in prostate cancer, therefore, the present study aimed to confirm this effect in $\mathrm{PCa}$.

PCa PC-3 cells were transfected with either an miR-494 overexpression (oe494) or negative control (scr) adenovirus, following which the mRNA and protein expression levels of survivin were determined using Western blot and RT-qPCR analyses. As shown in Fig. 2B, overexpression of miR-494 significantly decreased the protein expression of survivin in PC-3 cells. However, the mRNA expression of survivin was not affected by overexpression of miR-494 (Fig. 2C).

Taken together, the results (as shown in Fig. 2A-C) showed that miR-494 targeted survivin at the translational level in PCa.

miR-494 combined with survivin shRNA has synergistic effects on antiproliferation, inducing cell cycle arrest and cell apoptosis in PC-3 cells. The fact that miR-494 is decreased in $\mathrm{PCa}$ indicated that it may contribute to the progression of $\mathrm{PCa}$. Therefore, the present study examined the effect of miR-494 and survivin shRNA on cell growth, cell cycle arrest and apoptosis in PCa PC-3 cells. The PC-3 cells were transfected with either miR-494 (oe494) or survivin shRNA (shSur) or the two together (oe494+shSur). Their negative control groups were scr1, scr2 and scr1+scr2, respectively, and a PBS group served as a blank control.

Cell proliferation was measured using an MTT assay. The results indicated that all the three groups inhibited PC-3 cell proliferation, compared with their control groups. Notably, the oe494+shSur group had increased antiproliferation effects, compared with the oe494 or shSur groups alone (Fig. 3A).

Flow cytometry was performed to examine the cell cycle and cell apoptosis. The results showed that there was significant cell cycle arrest at the $\mathrm{G}_{2} / \mathrm{M}$ phase in the oe 494 , shSur and oe $494+$ shSur groups. The percentage of cells in the $\mathrm{G}_{2} / \mathrm{M}$ phase in these groups were $(8.19 \pm 3.23,34.56 \pm 4.06$ and $63.87 \pm 4.33 \%$, respectively (Fig. $3 \mathrm{~B}$ and C). Similarly, cell apoptosis, detected using Annexin-V/PI staining, showed that the percentages of cell apoptosis in these groups were $23.79 \pm 4.36,27.38 \pm 5.94$ and $49.98 \pm 5.85 \%$, respectively (Fig. 3D and E). 


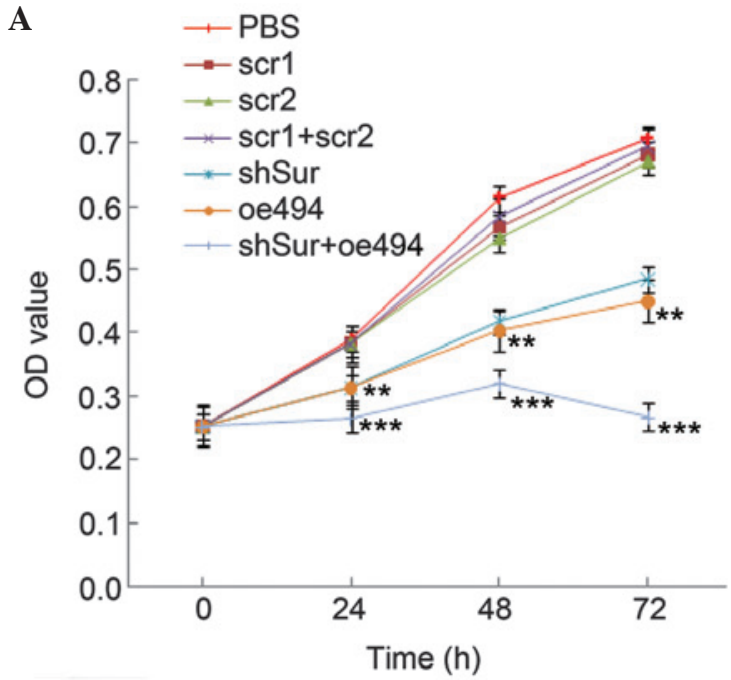

B

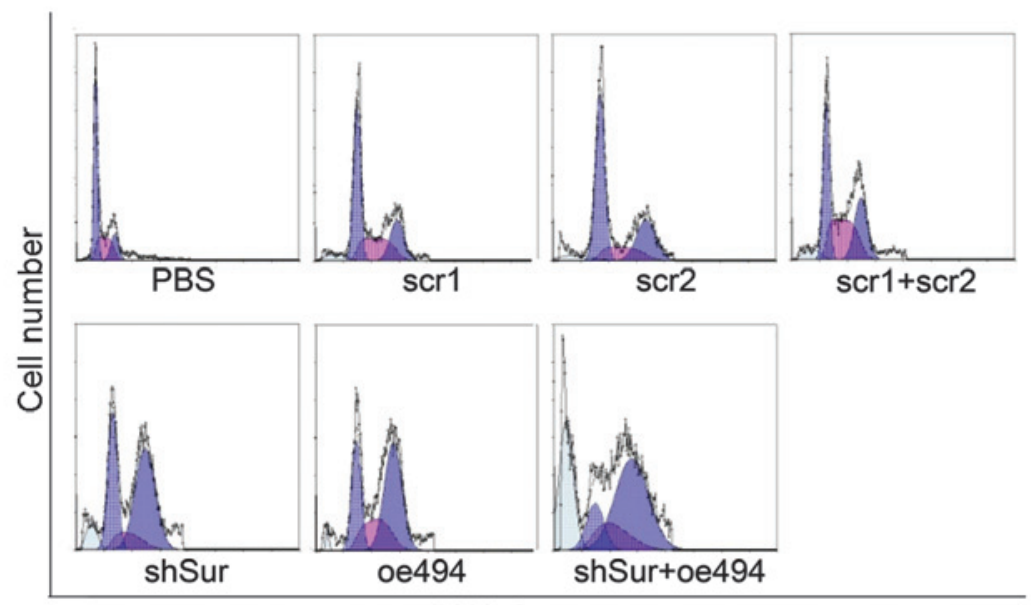

D

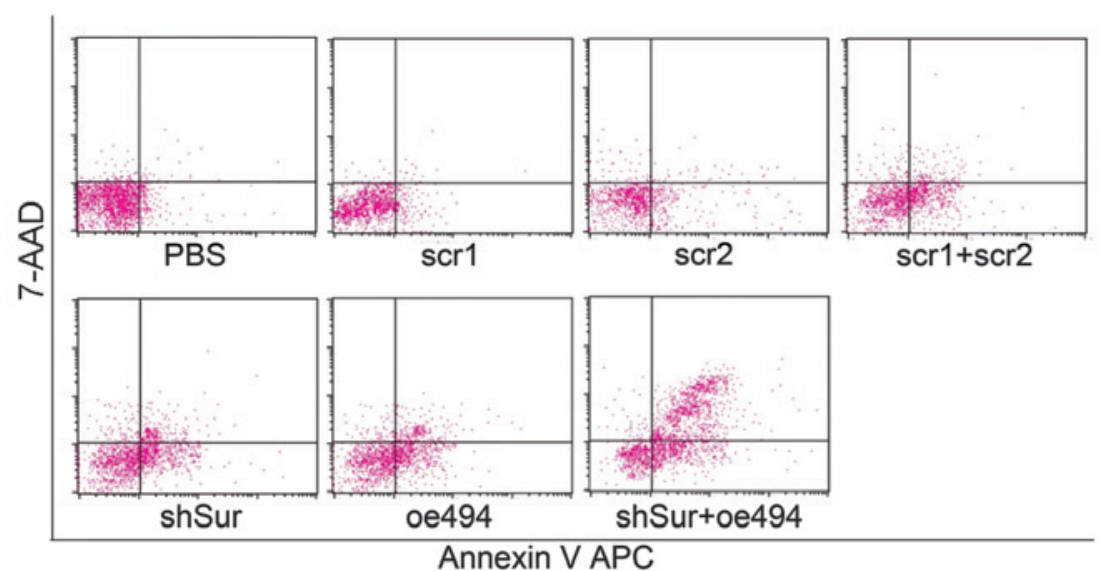

C

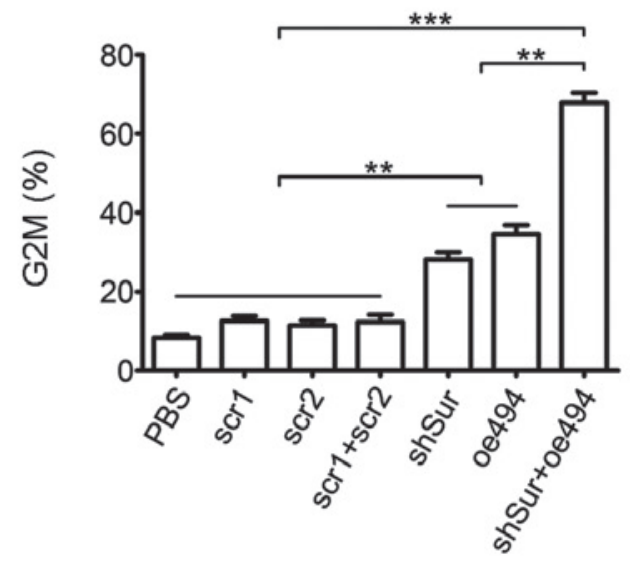

$\mathbf{E}$

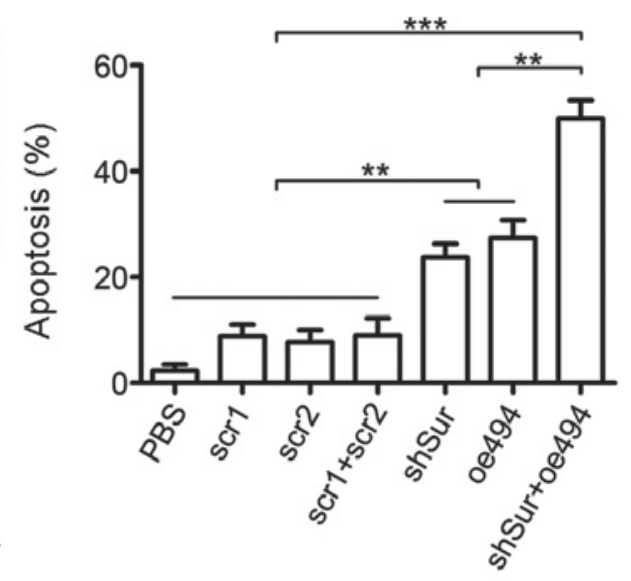

Figure 3. Synergistic effects of miR-494 combined with survivin shRNA on antiproliferation, induction of cell cycle arrest and apoptosis in PC-3 cells. The PC-3 cells were transfected with an adenovirus carrying either miR-494 or survivin shRNA, or both. (A) A 3-(4,5-dimethylthiazol-2-yl)-2,5-diphenyltetrazolium bromide assay was used to detect cell proliferation. Flow cytometry with PI staining or Annexin-V/PI staining were used to detect (B and C) cell cycle arrest and (D and E) apoptosis. Data are expressed as the mean \pm standard deviation. ${ }^{* *} \mathrm{P}<0.01,{ }^{* * *} \mathrm{P}<0.001$. miR, microRNA; shRNA, short hairpin RNA; PI, propidium iodide; PBS, phosphate-buffered saline; OD, optical density.

Taken together, the results (as shown in Fig. 2A-E) indicated that miR-494 and survivin shRNA were able to inhibit proliferation, and induce cell cycle arrest and cell apoptosis in PCa PC-3 cells. Of note, miR-494 combined with survivin shRNA had a more marked effect. These results indicated that targeting a single gene using two methods simultaneously had synergetic effects.

miR-494+survivin shRNA has a synergistic effect on inhibiting the protein expression of survivin in $P C a$. The present 
A

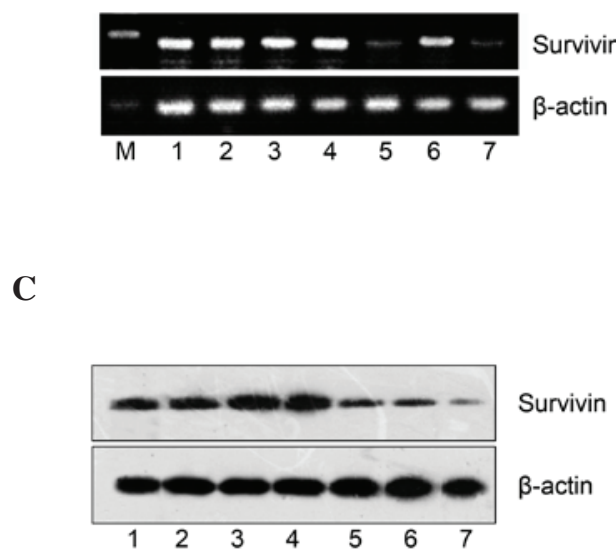

B

D
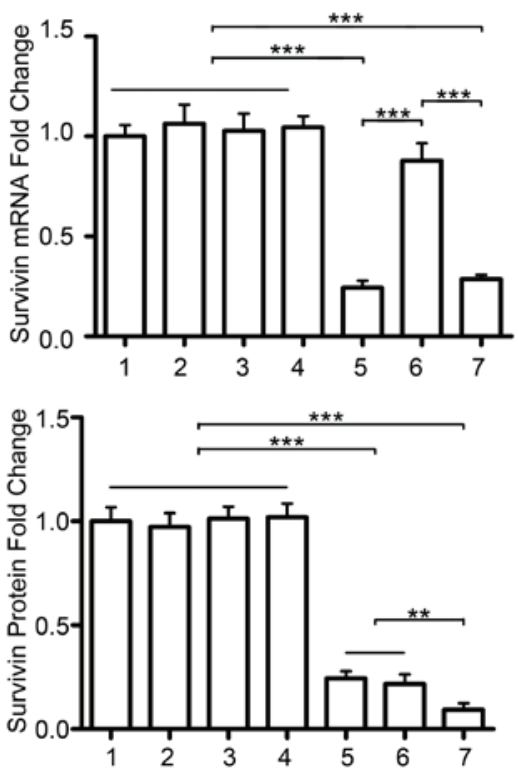

Figure 4. miR-494+survivin shRNA have a synergistic effect on inhibiting the protein expression of survivin in prostate cancer. (A) Reverse transcription-quantitative polymerase chain reaction analyses revealed that co-transfection with survivin shRNA and miR-494 did not further decrease the mRNA levels of survivin mRNA, compared with the group transfected with shRNA alone. (B) Quantitative data. (C) Transfection with miR-494 or survivin shRNA alone inhibited the protein expression of survivin in PC-3 cells, which were detected using Western blot analysis. However, the combination of the two had marked synergistic effects on the protein expression of survivin. (D) Quantitative data. 1, PBS; 2, scr1; 3, scr2; 4, scr1+scr2; 5, shSur; 6, oe494; 7, shSur+oe494. Data are expressed as the mean \pm standard deviation. ${ }^{* *} \mathrm{P}<0.01,{ }^{* * *} \mathrm{P}<0.001$. miR, microRNA; shRNA, short hairpin RNA; PBS, phosphate-buffered saline; M, marker.

study hypothesized that the marked effect of the combination of miR-494 and survivin shRNA is due to a further decrease in the protein expression of survivin. The PC-3 cells were transfected with either miR-494 (oe494) or survivin shRNA (shSur), or the two together (oe494+shSur). Although the mRNA expression of survivin remained unchanged in the oe494+shSur group, compared with the shSur group (Fig. 4A), the combination group had significantly lower protein levels of survivin, compared with either the oe494 group or the shSur group alone (Fig. 4B and C). This indicated that simultaneously suppressing survivin gene expression using different methods may have synergistic effects.

miR-494+survivin shRNA has a synergistic effect on PCa growth in vivo. As the in vitro data showed that either miR-494 or survivin shRNA effectively inhibited cell growth and induced cell apoptosis in PC-3 cells, and the combination of the two was more effective, the present study investigated whether the same effects were observed in vivo. A total of 25 male nude mice were divided into five groups: PBS, scrl, oe 494, shSur and oe $494+$ shSur. A total of $10^{7}$ treated cells were subcutaneously injected into the right axillary space of each nude mouse. Tumor size was monitored every 5 days and, 55 days post-injection, the mice were sacrificed, tumors were collected and tumor volume was calculated. The results demonstrated that tumors in the miR-494, survivin shRNA or the combined group were smaller, compared with those in the control (Fig. 5A and B). In addition, the combined group had a more marked effect on cancer growth, compared with the individual groups (Fig. 5A and B). Although the expression levels of survivin in the tumor tissues of all group were reduced, the expression was reduced most markedly in the combined group, detected using Western blot analysis and immunohistochemical staining (Fig 5C and D), which further confirmed the results derived from the in vitro experiments.

Taken together, these data indicated that miR-494 and survivin shRNA inhibited the expression of survivin and suppressed $\mathrm{PCa}$ cell growth in vitro and in vivo. Combining miR-494 with survivin shRNA had more significant effects on the suppressing the gene expression of survivin and $\mathrm{PCa}$ growth, compared with either the mir-494 or survivin shRNA treatment groups. The in vitro and in vivo data obtained in the present study confirmed that simultaneously suppressing the gene expression of survivin using different methods may have synergistic effects.

\section{Discussion}

The mechanisms involved in the carcinogenesis, progression and metastasis of PCa are complex. Substantial evidences has indicated that oncogenes, anti-oncogenes, microRNAs and long non-coding RNA are involved in PCa. However, their individual roles have been considered less important, than androgen receptor (AR), as AR target therapy, which constitutes the ADT strategy, is the mainstay for the treatment of advanced PCa. At present, no single oncogene or anti-oncogene target therapy has been found to be as effective as ADT for used to treat $\mathrm{PCa}$ in clinical settings. The primary reason for this is that the gene-based regulatory pathways are complex. For example, one gene can regulate the function of several downstream genes, and the gene itself is also controlled by multiple upstream genes. However, it is difficult to determine which oncogene or anti-oncogene is key in $\mathrm{PCa}$, particularly in the progression of CRPC.

The survivin gene, a member of the IAP family, has been confirmed to be overexpressed in almost all types of cancer 
A

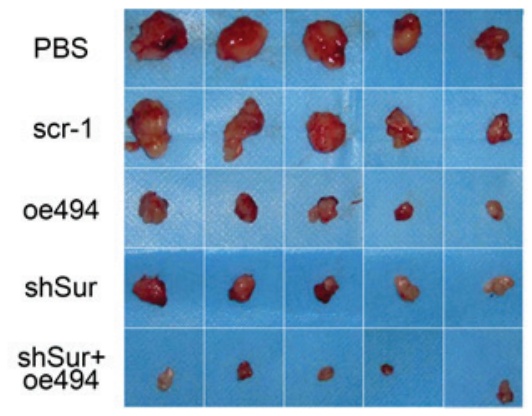

C

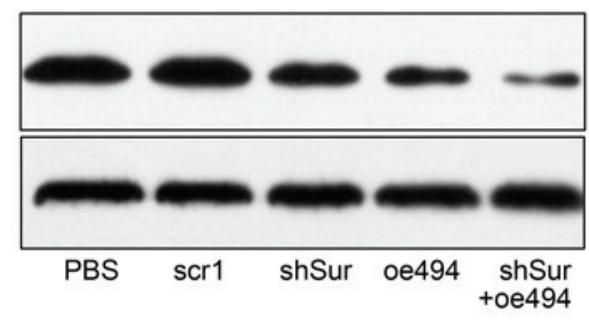

B
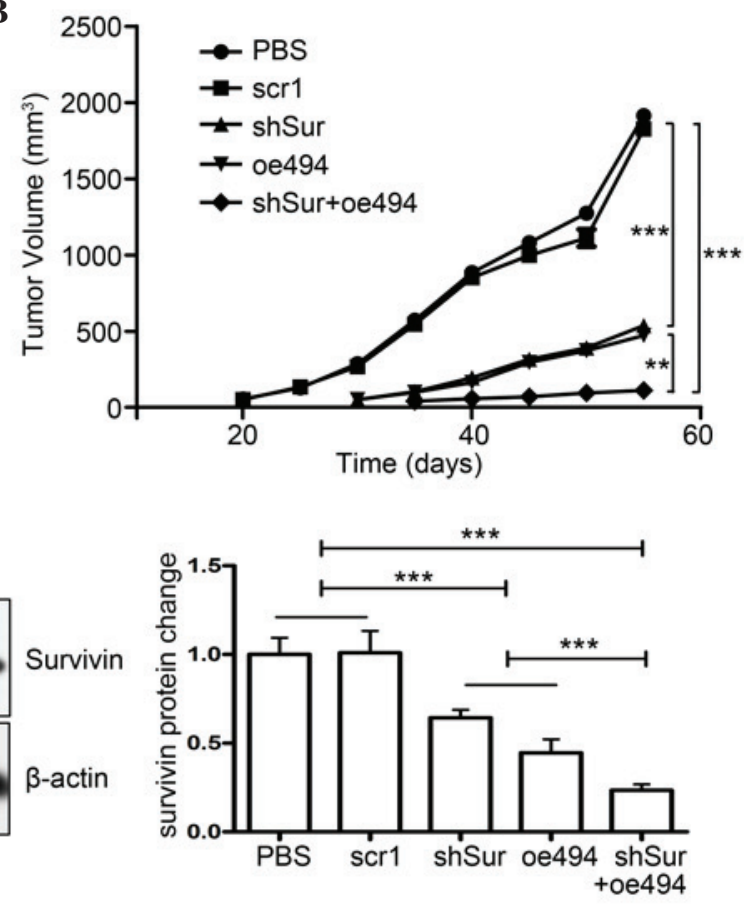

D

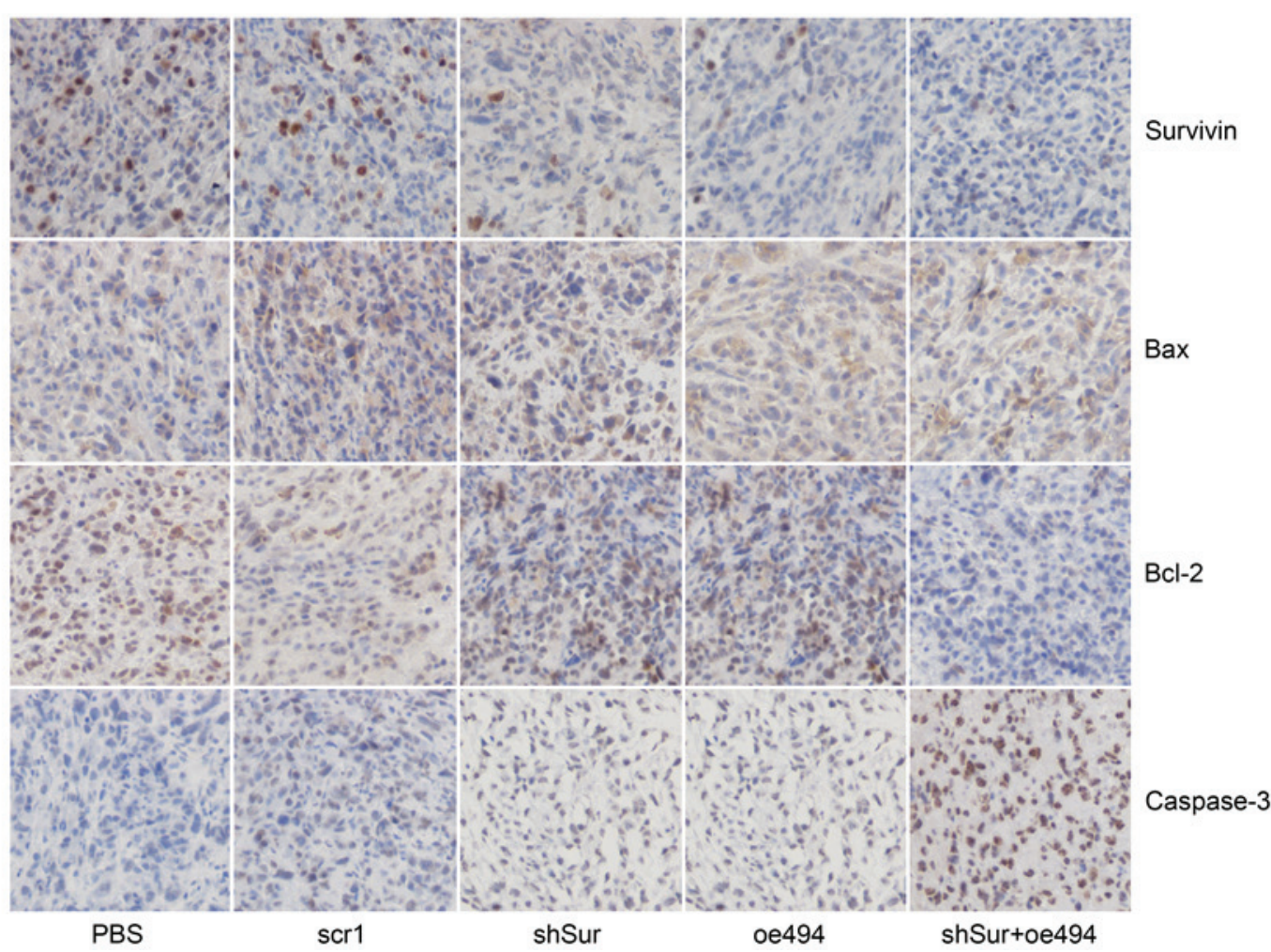

Figure 5. Synergistic effect of miR-494+survivin shRNA on PCa growth in vivo. PCa PC-3 cells were transfected with either miR-494 overexpression or survivin shRNA adenovirus or both, and were subcutaneously injected into 6-8-week-old male nude mice. Tumor size was monitored every 5 days and, 55 days post-injection, the mice were sacrificed and the tumors were harvested for evaluation. (A) Gross morphology of tumors in each group. (B) Tumor growth curve shows that, although miR-494 or survivin shRNA alone inhibited the growth of xenograft tumors, co-transfection with miR-494 and survivin shRNA had a synergistic effect, compared with either miR-494 or survivin shRNA alone $(\mathrm{P}<0.05)$. Expression levels of survivin in xenograft tumors were analyzed using (C) Western blot analysis and (D) immunohistochemical staining (magnification, $\mathrm{x} 200$ ) demonstrating that survivin and Bcl-2 expression levels were decreased and Bax and caspase- 3 increased in the shSur and oe 494 groups. Combination of shSur + oe 494 had synergistic effect on expression levels of these proteins. Cell nuclei are stained blue and proteins are stained brown. The results showed that miR-494, survivin shRNA or both inhibited the protein expression of survivin, and the combined group had synergetic effects $(\mathrm{P}<0.05)$. Data are expressed as the mean \pm standard deviation. ${ }^{* *} \mathrm{P}<0.01{ }^{* * * *} \mathrm{P}<0.001$. $\mathrm{PCa}$, prostate cancer; miR, microRNA; shRNA, short hairpin RNA; PBS, phosphate-buffered saline; M, marker; Bcl-2, B-cell lymphoma 2; Bax, BCL2-associated X protein.

cell, which include radiation resistant $(10,21,22)$ and drug resistant (23-25) cancer cells, as well as CRPC cells $(26,27)$.
Inhibiting the gene expression of survivin suppresses $\mathrm{PCa}$ cell growth, induces apoptosis and enhances radiation and 
drug sensitivity in PCa cells, as well as in other types of cancer cell (28). These findings indicate that survivin may be a potential useful target for anticancer intervention. A number of anti-survivin strategies, including the use of the antisense oligonucleotide, LY2181308 (27), small interfering (si)RNA (29) and locked nucleic acid siRNA-based strategies (30) have been reported to successfully reduce the expression of survivin, inducing cell apoptosis and enhancing chemosensitivity in various types of cancer in vitro, which include CRPC (31). However, these strategies have only yielded a partial positive response in clinical trials, which may be due to the inhibition of survivin by a single agent being insufficient Although the reduction in the protein expression of survivin has been reported to be at least $30 \%$ upon the treatment with LY2181308, to achieve a more marked effect, $>30 \%$ inhibition from baseline is required (27). This suggests that directly knocking down survivin via one method has only a limited effect in suppressing cancer proliferation.

As cancer cell proliferation is regulated by multiple genes, whether knocking down two oncogenes simultaneously or knocking down one oncogene combined with the overexpression of another anti-oncogene has more marked effects has been investigated. For example, simultaneously knocking down survivin and vascular endothelial growth factor has shown synergistic effects on inhibiting in vitro cell proliferation and in vivo tumor growth in pancreatic cancer cell (32). In addition, survivin knockdown combined with apoptin over-expression inhibits cell growth significantly in HeLa cells and HepG2 cells (33). The co-expression of survivin-specific siRNA and wild-type p53 have also been observed to significantly inhibit PCa cell proliferation in vitro and in vivo (34).

These previous reports indicate that the effects of simultaneously controlling the expression of two genes are more marked, compared with the effect of controlling one individual gene for suppressing cancer cell growth. As one target gene is controlled by multiple mechanisms, including DNA amplification, mRNA translation and protein modification (35), whether inhibiting one gene via two methods has more advanced effects remains to be fully elucidated.

Our bioinformatics analysis and experimental results confirmed that miR-494 targets survivin in PCa. This is consistent with a previous report that miR-494 induces cell apoptosis by suppressing the gene expression of survivin in AML cells (18). Various reports have also shown that miR-494 is downregulated in multiple types of cancer, including liver cancer (36) and pancreatic cancer, as well as in PCa (13). Furthermore, miR-494 inhibits cell proliferation and induces cell apoptosis by regulating the expression of multiple genes, including KIT (17), BIM (16), C-X-C chemokine receptor type 4 (37) and survivin (18). The present study investigated the role of miR-494 and its interaction with survivin in PCa growth. The results indicated that miR-494 was decreased in PCa tissues and in the PC-3 cell line. Overexpression of miR-494 was found to inhibit cell proliferation and induce cell apoptosis in PC-3 cells by inhibiting the expression of survivin, and its activity is similar to that of survivin shRNA. Notably, simultaneous transfection with miR-494 and survivin shRNA had synergistic effects on the expression of survivin and on the growth of the PC-3 cells in vitro and in vivo.

\section{Acknowledgements}

The present study was supported by the National Natural Science Foundation of China (grant no. 81472776) for Professor D Yang, Dr J Zhu and Professor Y Shan, and the Natural Science Foundation of Jiangsu Province (grant no. BK2008167) for Professor D Yang and Professor Y Shan. It was also funded by the Priority Academic Program Development of Jiangsu Higher Education Institutes for Dr J Zhu, Professor Y Shan and Professor D Yang, the Specialized Research Fund for the Doctoral Program of Higher Education (grant no. 20133201110016) for Professor Y Shan, Professor D Yang, Dr J Zhu and Dr Y Zang, and the Preponderant Discipline Construction Funding of the Second Affiliated Hospital of Soochow University.

\section{References}

1. Oliver SE, Gunnell D and Donovan JL: Comparison of trends in prostate cancer mortality in England and Wales and the USA. Lancet 355: 1788-1789, 2000.

2. Zhang M, Latham DE, Delaney MA and Chakravarti A: Survivin mediates resistance to antiandrogen therapy in prostate cancer. Oncogene 24: 2474-2482, 2005.

3. Krajewska M, Krajewski S, Banares S, Huang X, Turner B, Bubendorf L, Kallioniemi OP, Shabaik A, Vitiello A, Peehl D, et al: Elevated expression of inhibitor of apoptosis proteins in prostate cancer. Clin Cancer Res 9: 4914-4925, 2003.

4. Shariat SF, Lotan Y, Saboorian H, Khoddami SM, Roehrborn CG, Slawin KM and Ashfaq R: Survivin expression is associated with features of biologically aggressive prostate carcinoma. Cancer 100: 751-757, 2004.

5. Ambrosini G, Adida C and Altieri DC: A novel anti-apoptosis gene, survivin, expressed in cancer and lymphoma. Nat Med 3: 917-921, 1997.

6. Ito T, Shiraki K, Sugimoto K, Yamanaka T, Fujikawa K, Ito M, Takase K, Moriyama M, Kawano H, Hayashida M, et al: Survivin promotes cell proliferation in human hepatocellular carcinoma. Hepatology 31: 1080-1085, 2000.

7. Sui L, Dong Y, Ohno M, Watanabe Y, Sugimoto K and Tokuda M: Survivin expression and its correlation with cell proliferation and prognosis in epithelial ovarian tumors. Int J Oncol 21: 315-320, 2002.

8. Zaffaroni N, Pennati M, Colella G, Perego P, Supino R, Gatti L, Pilotti S, Zunino F and Daidone MG: Expression of the anti-apoptotic gene survivin correlates with taxol resistance in human ovarian cancer. Cell Mol Life Sci 59: 1406-1412, 2002.

9. Lu B, Mu Y, Cao C, Zeng F, Schneider S, Tan J, Price J, Chen J, Freeman M and Hallahan DE: Survivin as a therapeutic target for radiation sensitization in lung cancer. Cancer Res 64: 2840-2845, 2004

10. Kennedy SM, O'Driscoll L, Purcell R, Fitz-Simons N, McDermott EW, Hill AD, O'Higgins NJ, Parkinson M, Linehan R and Clynes M: Prognostic importance of survivin in breast cancer. Br J Cancer 88: 1077-1083, 2003.

11. Kishi H, Igawa M, Kikuno N, Yoshino T, Urakami S and Shiina H: Expression of the survivin gene in prostate cancer: Correlation with clinicopathological characteristics, proliferative activity and apoptosis. J Urol 171: 1855-1860, 2004.

12. Zhang M, Mukherjee N, Bermudez RS, Latham DE, Delaney MA, Zietman AL, Shipley WU and Chakravarti A: Adenovirus-mediated inhibition of survivin expression sensitizes human prostate cancer cells to paclitaxel in vitro and in vivo. Prostate 64: 293-302, 2005.

13. Hayashi N, Asano K, Suzuki H, Yamamoto T, Tanigawa N, Egawa $\mathrm{S}$ and Manome Y: Adenoviral infection of survivin antisense sensitizes prostate cancer cells to etoposide in vivo. Prostate 65: 10-19, 2005

14. Jiang S, Zhang HW, Lu MH, He XH, Li Y, Gu H, Liu MF and Wang ED: MicroRNA-155 functions as an OncomiR in breast cancer by targeting the suppressor of cytokine signaling 1 gene. Cancer Res 70: 3119-3127, 2010. 
15. Liu C, Kelnar K, Liu B, Chen X, Calhoun-Davis T, Li H, Patrawala L, Yan H, Jeter C, Honorio S, et al: The microRNA miR-34a inhibits prostate cancer stem cells and metastasis by directly repressing CD44. Nat Med 17: 211-215, 2011.

16. Romano G, Acunzo M, Garofalo M, Di Leva G, Cascione L, Zanca C, Bolon B, Condorelli G and Croce CM: MiR-494 is regulated by ERK1/2 and modulates TRAIL-induced apoptosis in non-small-cell lung cancer through BIM down-regulation. Proc Natl Acad Sci USA 109: 16570-16575, 2012.

17. Kim WK, Park M, Kim YK, Tae YK, Yang HK, Lee JM and Kim H: MicroRNA-494 downregulates KIT and inhibits gastrointestinal stromal tumor cell proliferation. Clin Cancer Res 17: 7584-7594, 2011.

18. Diakos C, Zhong S, Xiao Y, Zhou M, Vasconcelos GM, Krapf G, Yeh RF, Zheng S, Kang $M$ and Wiencke JK: TEL-AML1 regulation of survivin and apoptosis via miRNA-494 and miRNA-320a. Blood 116: 4885-4893, 2010.

19. Chang SS, Jiang WW, Smith I, Poeta LM, Begum S, Glazer C, Shan S, Westra W, Sidransky D and Califano JA: MicroRNA alterations in head and neck squamous cell carcinoma. Int J Cancer 123: 2791-2797, 2008.

20. Ambs S, Prueitt RL, Yi M, Hudson RS, Howe TM, Petrocca F, Wallace TA, Liu CG, Volinia S, Calin GA, et al: Genomic profiling of microRNA and messenger RNA reveals deregulated microRNA expression in prostate cancer. Cancer Res 68 6162-6170, 2008

21. Cao C, Mu Y, Hallahan DE and Lu B: XIAP and survivin as therapeutic targets for radiation sensitization in preclinical models of lung cancer. Oncogene 23: 7047-7052, 2004.

22. Chakravarti A, Zhai GG, Zhang M, Malhotra R, Latham DE, Delaney MA, Robe P, Nestler U, Song Q and Loeffler J: Survivin enhances radiation resistance in primary human glioblastoma cells via caspase-independent mechanisms. Oncogene 23: 7494-7506, 2004.

23. Olie RA, Simões-Wüst AP, Baumann B, Leech SH, Fabbro D, Stahel RA and Zangemeister-Wittke U: A novel antisense oligonucleotide targeting survivin expression induces apoptosis and sensitizes lung cancer cells to chemotherapy. Cancer Res 60 : 2805-2809, 2000.

24. Kato J, Kuwabara Y, Mitani M, Shinoda N, Sato A, Toyama T, Mitsui A, Nishiwaki T, Moriyama S, Kudo J and Fujii Y: Expression of survivin in esophageal cancer: Correlation with the prognosis and response to chemotherapy. Int J Cancer 95 92-95, 2001.

25. Als AB, Dyrskjøt L, von der Maase H, Koed K, Mansilla F Toldbod HE, Jensen JL, Ulhøi BP, Sengeløv L, Jensen KM and Orntoft TF: Emmprin and survivin predict response and survival following cisplatin-containing chemotherapy in patients with advanced bladder cancer. Clin Cancer Res 13: 4407-4414, 2007.
26. Tolcher AW, Quinn DI, Ferrari A, Ahmann F, Giaccone G, Drake T, Keating A and de Bono JS: A phase II study of YM155, a novel small-molecule suppressor of survivin, in castration-resistant taxane-pretreated prostate cancer. Ann Oncol 23: 968-973, 2012.

27. Wiechno P, Somer BG, Mellado B, Chłosta PL, Cervera Grau JM, Castellano D, Reuter C, Stöckle M, Kamradt J, Pikiel J, et al: A randomised phase 2 study combining LY2181308 sodium (survivin antisense oligonucleotide) with first-line docetaxel/prednisone in patients with castration-resistant prostate cancer. Eur Urol 65: 516-520, 2014.

28. Altieri DC: Validating survivin as a cancer therapeutic target. Nat Rev Cancer 3: 46-54, 2003.

29. Ning S, Fuessel S, Kotzsch M, Kraemer K, Kappler M, Schmidt U, Taubert H, Wirth MP and Meye A: siRNA-mediated down-regulation of survivin inhibits bladder cancer cell growth. Int J Oncol 25: 1065-1071, 2004.

30. Sapra P, Wang M, Bandaru R, Zhao H, Greenberger LM and Horak ID: Down-modulation of survivin expression and inhibition of tumor growth in vivo by EZN-3042, a locked nucleic acid antisense oligonucleotide. Nucleosides Nucleotides Nucleic Acids 29: 97-112, 2010.

31. Wiechno P, Chlosta P, Smok-Kalwat J, Pikilel J, Henry D, Christianson D, Somer B, Mellado B, Duran I and Castellano D: Interim results of a randomized phase II study with window-design to evaluate antitumor activity of the survivin antisense oligonucleotide (ASO) LY2181308 in combination with docetaxel for first-line treatment of castrate-resistant prostate cancer (CRPC). J Clin Oncol 29: (suppl; abstr 4592), 2011.

32. Song J, Cao L and Li Y: RNA interference-mediated inhibition of survivin and VEGF in pancreatic cancer cells in vitro. Mol Med Rep 7: 1651-1655, 2013.

33. Liu Q, Fu H, Xing R, Tie Y, Zhu J, Sun Z and Zheng X: Survivin knockdown combined with apoptin overexpression inhibits cell growth significantly. Cancer Biol Ther 7: 1053-1060, 2008.

34. Shao Y, Liu Y, Shao C, Hu J, Li X, Li F, Zhang L, Zhao D, Sun L, Zhao $\mathrm{X}$, et al: Enhanced tumor suppression in vitro and in vivo by co-expression of survivin-specific siRNA and wild-type p53 protein. Cancer Gene Ther 17: 844-854, 2010.

35. Baron M, Aslam H, Flasza M, Fostier M, Higgs JE, Mazaleyrat SL and Wilkin MB: Multiple levels of Notch signal regulation. Mol Membr Biol 19: 27-38, 2002.

36. Huang YS, Dai Y, Yu XF, Bao SY, Yin YB, Tang M and Hu CX: Microarray analysis of microRNA expression in hepatocellular carcinoma and non-tumorous tissues without viral hepatitis. J Gastroenterol Hepatol 23: 87-94, 2008.

37. Shen PF, Chen XQ, Liao YC, Chen N, Zhou Q, Wei Q, Li X, Wang J and Zeng H: MicroRNA-494-3p targets CXCR4 to suppress the proliferation, invasion and migration of prostate cancer. Prostate 74: 756-767, 2014. 